DOI: https://doi.org/10.11144/Javeriana.upsy16-2.evvi

\title{
Evidencias de validez de la versión en español del Inventario de Fobia Social (SPIN) ${ }^{*}$
}

Evidence of validity of the spanish version of the Social Phobia Inventory (SPIN)

\author{
Marcelo-Archibaldo Bravo \\ Universidad Michoacana de San Nicolás de Hidalgo, México \\ ORCID: http://orcid.org/0000-0002-6940-0382 \\ Fabiola González Betanzos \\ Universidad Michoacana de San Nicolás de Hidalgo, México \\ Álvaro Castillo Navarro \\ Instituto Mexicano del Seguro Social, México \\ Ferrán Padrós Blázquez \\ Universidad Michoacana de San Nicolás de Hidalgo, México
}

\footnotetext{
a Autor de correspondencia. Correo electrónico: archibaldo.bravo@yahoo.com

Para citar este artículo: Bravo, M. A., González Bentazos, F., Castillo Navarro, A., \& Padrós Blázquez, F. (2017). Evidencias de validez de la versión en español del Inventario de Fobia Social (SPIN). Universitas Psychologica, 16(2), 1-12.

https://doi.org/10.11144/Javeriana.upsy16-2.evvi
}

\section{RESUMEN}

El objetivo del presente estudio fue analizar en muestras mexicanas las propiedades psicométricas del Inventario de Fobia Social (Social Phobia Inventory, SPIN). Los participantes consistieron en estudiantes universitarios, pacientes diagnosticados con trastorno de ansiedad social y pacientes con trastorno de ansiedad generalizada. El SPIN mostró una alta consistencia interna, una buena confiabilidad test-retest y una adecuada validez convergente y discriminante. Al analizar la estructura interna, el modelo que manifestó mejores indicadores de ajuste fue el de tres factores correlacionados. El análisis de curvas ROC indicó que el SPIN es capaz de discriminar entre los trastornos de ansiedad social y de ansiedad generalizada; un punto de corte de 25 representó el mejor balance entre sensibilidad y especificidad. Se concluye que la versión en español del SPIN demuestra propiedades psicométricas sólidas, por lo que puede ser potencialmente utilizado como instrumento de cribado para detectar el trastorno de ansiedad social en población mexicana.

\section{Palabras clave}

trastorno de ansiedad social; fobia social; evaluación; psicometría; SPIN

\begin{abstract}
The aim of this study was to analyze the psychometric properties of the Social Phobia Inventory (SPIN), in Mexican samples. The participants comprised university students, patients diagnosed with social anxiety and patients with generalized anxiety disorder. The SPIN demonstrated high internal consistency, good test-retest reliability, and adequate convergent and discriminate validity. Different models of the internal structure were tested, result showed better adjustment for the three correlated factor model. Analysis of ROC curves indicated that the SPIN is able to discriminate between social anxiety and generalized anxiety disorders; a cutoff of 25 represented the best balance between sensitivity and
\end{abstract}


specificity. It is possible to conclude that the Spanish version of the SPIN demonstrated solid psychometric properties, and thus it could potentially be used as a screening tool for detecting social anxiety disorder in the Mexican population.

Keywords

social anxiety disorder; social phobia; evaluation; psychometrics; SPIN

\section{Introducción}

El trastorno de ansiedad social (fobia social) consiste en un miedo intenso a una o más situaciones sociales, donde el individuo está expuesto a la probable evaluación de otras personas y teme mostrar síntomas de ansiedad que sean valorados negativamente (American Psychiatric Association, 2013). La revisión de Somers, Goldner, Waraich, y Hsu (2006) indica que este trastorno tiene una prevalencia a lo largo de la vida de entre 0.53 a $16 \%$, al tomar en cuenta datos de diferentes países. Específicamente, en México, se han encontrado cifras que van desde 2.6 (Caraveo \& Colmenares, 2000) a 4.7 \% (Medina-Mora, et al., 2003).

La ansiedad social está considerada entre los trastornos de ansiedad más frecuentes (Bernaldode-Quirós, et al., 2012; Somers, et al., 2006), y de acuerdo con diversos estudios (Acarturk, Graaf, Straten, Have, \& Cuijpers., 2008; Aderka, et al., 2012; Furmark, 2002; Simon, et al., 2002; Torgrud, et al., 2004; Wong, Sarver, \& Beidel, 2012), la presencia de este está asociada a un deterioro en la calidad de vida, con una baja tasa de apoyo social y con dificultades académicas, laborales y sociales. Además, la ansiedad social se asocia frecuentemente a otros padecimientos, siendo los trastornos comórbidos más comunes, otros trastornos de ansiedad, del estado de ánimo y de abuso de sustancias (Acarturk, et al., 2008; Chartier, Walker \& Stein, 2003; Ohayon \& Schatzberg, 2010).

Es importante destacar que habitualmente la edad de inicio de la ansiedad social precede a la del trastorno comórbido (Chartier et al., 2003). Dado que es una condición potencialmente tratable (véase Labrador \& Ballesteros, 2011), su identificación temprana puede ayudar a prevenir estas complicaciones. Por lo que es fundamental contar con instrumentos adecuados que evalúen la presencia y gravedad de este trastorno, tanto para la detección de casos como para la evaluación de la eficacia de los tratamientos empleados.

Uno de los instrumentos de cribado más utilizados en la práctica clínica en países anglosajones es el Inventario de Fobia Social (Social Phobia Inventory, SPIN; Connor, et al., 2000). Este instrumento evalúa los diferentes aspectos asociados al espectro de la ansiedad social (i. e. síntomas conductuales, fisiológicos y cognitivos). Asimismo, ha demostrado, en muestras de distintos países, una alta consistencia interna, una adecuada confiabilidad test-retest y evidencias sólidas de validez convergente y discriminante (Antony, Coons, McCabe, Ashbaugh, \& Swinson, 2006; García-López, Bermejo, \& Dolores-Hidalgo, 2010; Radomsky et al., 2006; Ranta, et al., 2007; Sosic, Gieler, \& Stangier, 2008).

Además de los Estados Unidos, el SPIN ha sido validado en Canadá (Antony, et al., 2006; Radomsky et al., 2006), Finlandia (Ranta et al., 2007), Alemania (Sosic et al., 2008), Brasil (Osório, Crippa, \& Loureiro, 2009; Vilete, Coutinho, \& Figueira, 2004) y España (GarcíaLópez et al., 2010). Las principales ventajas de este instrumento son: 1) Permite distinguir entre personas con trastorno de ansiedad social de aquellas que no lo padecen, a partir del establecimiento de puntos de corte. 2) Es una medida que permite identificar la severidad de los síntomas de la ansiedad social. 3) Tanto la puntuación total como las puntuaciones de las subescalas han demostrado ser sensibles a los cambios clínicos debidos a tratamientos farmacológicos o psicoterapéuticos (Allgulander et al., 2004; Antony et al., 2006; Rickels, Mangano, \& Khan, 2004; Stein, Versiani, Hair, \& Kumar, 2002).

De esta manera, el propósito de la presente investigación es analizar, en muestras mexicanas, las propiedades psicométricas de la versión en español del Inventario de Fobia Social (Social Phobia Inventory, SPIN; Connor, et al., 2000). Los objetivos del estudio son: 1) Proporcionar la media y desviación estándar 
del SPIN en cada una de las muestras estudiadas (estudiantes universitarios, pacientes con trastorno de ansiedad social y pacientes con trastorno de ansiedad generalizada). 2) Analizar la estructura interna. 3) Evaluar la consistencia interna. 4) Estudiar la confiabilidad test-retest. 5) Comprobar la validez convergente y discriminante. 6) Analizar el poder discriminativo del SPIN para diferenciar entre los trastornos de ansiedad social y ansiedad generalizada, a partir de un análisis de curvas ROC.

\section{Método}

\section{Participantes}

El número total de participantes fue de 998 sujetos, divididos en 4 muestras (véase Tabla 1). Las muestras "A" $(n=530)$ y "B" $(n=$ 367) estuvieron formadas por estudiantes de la Universidad Michoacana San Nicolás de Hidalgo (México). Dichas muestras, se obtuvieron a partir de los mismos grupos de alumnos, pero en dos momentos distintos (6 semanas de diferencia). Los alumnos que aceptaron participar en ambas aplicaciones fueron 276 alumnos, y con ellos se estudió la estabilidad temporal del instrumento (confiabilidad test-retest).

La muestra "C" estuvo conformada por pacientes diagnosticados con trastorno de ansiedad social $(n=51)$ y la "D" $(n=50)$ por individuos con diagnóstico de trastorno de ansiedad generalizada. Estas muestras fueron recolectadas por un médico psiquiatra, a través del área de consulta externa del Instituto Mexicano del Seguro Social (Hospital General de Zona N.. 83, Morelia), de acuerdo con los criterios diagnósticos del DSM-5 (American Psychological Association, 2013).

\section{Instrumentos}

Inventario de Fobia Social (Social Phobia Inventory, SPIN). El SPIN fue desarrollado por Connor, et al. (2000) basándose en la Escala Breve de
Fobia Social (Davidson, et al. 1991). Cuenta con 17 ítems, que se contestan en una escala de 5 puntos (de 0 a 4). La puntuación media para sujetos con trastorno de ansiedad social es de $41.1(D E=10.2)$ y para individuos sin ansiedad social es de $12.1(D E=9.3)$. Las puntuaciones de corte que han sido propuestas para distinguir a personas con ansiedad social de las que no presentan el trastorno, son de 19 en sujetos estadounidenses (Connor, et al., 2000), de 21 en población española (García-López, et al., 2010) y de 24 en adolescentes finlandeses (Ranta, et al., 2007). En la publicación original, el SPIN presentó una consistencia interna de 0.87 a 0.94 en sujetos con ansiedad social y de 0.82 a 0.90 en personas sin ella, así como una confiabilidad test-retest (evaluada a tres semanas) de 0.84 a 0.78 en personas con ansiedad social (Connor, et al., 2000), además de demostrar ser sensible al cambio después de un tratamiento.

Escala de Miedo a la Evaluación Negativa version breve (Brief version of the Fear of Negative Evaluation Scale, BFNE). Esta escala fue desarrollada por Leary (1983), y consiste en una versión breve de la Escala de Miedo a la Evaluación Negativa (FNE; Watson \& Friend, 1969). Cuenta con 12 ítems que se contestan en una escala de 5 puntos (de 1 a 5), 4 de los cuales se califican de manera inversa $(2,4$, 7 y 10). Las propiedades psicométricas de esta escala han sido evaluadas en muestras mexicanas (Bravo, González, Castillo, \& Padrós, 2015), en donde se ha reportado una consistencia interna de .89 , así como una puntuación media de 30.44 $(D E=6.62)$ en estudiantes universitarios y de $52.16(D E=2.71)$ en personas con trastorno de ansiedad social.

Escala de Ansiedad ante la Interacción Social (Social Interaction Anxiety Scale, SIAS). Este instrumento fue desarrollado por Mattick y Clarke (1998) y cuenta con 20 ítems que se contestan en una escala de 5 puntos (de 0 a 4), 3 de los cuales se califican de manera inversa (5, 9 y 11). Presenta una media de 24.6 (DE $=16.4$ ) en individuos con ansiedad social y de $18.8(\mathrm{DE}=11.8)$ en sujetos sin problemas de ansiedad. La consistencia interna es de 0.86 a 
0.94 y la confiabilidad test-retest de 0.86 a 0.92 en un periodo de cuatro a doce semanas.

Escala de Fobia Social (Social Phobia Scale, SPS). Desarrollado por Mattick y Clarke (1998), es una escala que posee 20 ítems que se califican en una escala de 5 puntos (de 0 a 4). Los sujetos con ansiedad social han obtenido una puntuación media de $40(D E=16)$ y las personas sin problemas de ansiedad una media de 14.4 (DE $=11.2$ ). Su consistencia interna ha sido de 0.87 a 0.94 y la confiabilidad test-retest (en un periodo de cuatro a doce semanas) de 0.66 a 0.93 .

Inventario de Ansiedad de Beck (Beck Anxiety Inventory, BAI). Consta de 21 ítems que se contestan en una escala de 4 puntos (de 0 a 3 ), los cuales miden síntomas de ansiedad general. En esta investigación, se utilizó la versión mexicana adaptada por Robles, Varela, Jurado y Páez (2001). La puntuación media para población general fue de $12(D E=9.3)$, para pacientes con trastornos de ansiedad de $36.9(D E=12.6)$ y para sujetos sanos de $9.7(\mathrm{DE}=7.0)$. Presentó una consistencia interna de 0.84 en estudiantes universitarios y de 0.83 en población general, así como una confiabilidad test-retest de 0.75 .

Inventario de Depresión de Beck (Beck Depresion Inventory, BDI). Se compone de 21 ítems (y un adicional), en cada uno de los cuales se tiene que elegir entre cuatro enunciados que describen el estado de la persona, lo cual viene siendo una escala de 4 puntos (de 0 a 3). Fue empleada la versión mexicana adaptada por Jurado et al. (1998), en la cual se obtuvo una consistencia interna de 0.87 en población general.

\section{Procedimiento}

En este estudio, se utilizó la traducción española del SPIN, disponible en García-Portilla et al. (2008). Este instrumento fue revisado por tres jueces con experiencia en el ámbito de la psicometría y con conocimientos sobre el trastorno de ansiedad social; ninguno de los jueces consideró pertinente la modificación de los ítems.

Para formar la muestra "A" $(n=530)$ de participantes, se asistió a grupos de diferentes licenciaturas de la Universidad Michoacana de San Nicolás de Hidalgo. Se explicó a los alumnos el objetivo de la investigación y se les invito a participar en la misma. Todos aquellos que aceptaron participar firmaron un formato de consentimiento informado, y les fueron aplicados los siguientes instrumentos: SPIN, BFNE, BAI y SIAS.

La muestra "B" $(n=367)$ fue formada también con estudiantes universitarios. Se acudió a los mismos grupos de estudiantes, y se hizo una segunda invitación para participar en el estudio; la evaluación se llevó a cabo seis semanas después de la primera aplicación. En esta ocasión, los instrumentos contestados fueron estos: SPIN, BDI y SPS. Únicamente 276 estudiantes respondieron en ambas ocasiones al SPIN.

Las muestras clínicas ("C" y "D") se recolectaron después de que el proyecto fuera aprobado por el Comité de Ética del Instituto Mexicano del Seguro Social (IMSS). Todos los pacientes que participaron dieron su consentimiento informado por escrito; el criterio de inclusión fue cumplir con los criterios diagnósticos del DSM-5 (American Psychological Association [APA], 2013) para la presencia de un trastorno de ansiedad social o de un trastorno de ansiedad generalizada.

\section{Resultados}

\section{Datos normativos}

La media de la puntuación total del SPIN en las muestras de estudiantes fue de $13.80(\mathrm{DE}=$ 9.12) y de $12.63(D E=9.48)$. En tanto que la media para sujetos con trastorno de ansiedad social fue de $52.39(D E=4.07)$ y para los individuos con ansiedad generalizada de 24.02 $(D E=8.71)$. En la Tabla 1, pueden observarse las medias, desviaciones estándar y rangos de las muestras "A", "B", "C" y "D", tanto en lo que se refiere a la puntuación total del SPIN como a sus tres subescalas (miedo, evitación y síntomas fisiológicos). 


\section{TABLA 1}

Estadísticos descriptivos de la puntuación total del SPIN y de sus tres subescalas en cada una de las muestras

\begin{tabular}{ccccc} 
& $\begin{array}{c}\mathbf{A} \\
\text { (universitarios } \\
1^{\circ} \text { aplicación) } \\
n=530\end{array}$ & $\begin{array}{c}\mathbf{B} \\
\text { (universitarios } \\
2^{\circ} \text { aplicación) } \\
n=367\end{array}$ & $\begin{array}{c}\mathbf{C} \\
\text { (ansiedad } \\
\text { social) } \\
n=51\end{array}$ & $\begin{array}{c}\mathbf{D} \\
\text { (ansiedad } \\
\text { generalizada) } \\
n=50\end{array}$ \\
\hline SPIN Total & & & & \\
Media & 13.80 & 12.63 & 52.39 & 24.02 \\
Desviación estándar & 9.12 & 9.48 & 4.07 & 8.71 \\
$\quad$ Rango & $0-57$ & $0-60$ & $45-64$ & $11-48$ \\
SPIN miedo & & & & \\
$\quad$ Media & 4.62 & 4.21 & 18.75 & 8.48 \\
Desviación estándar & 3.73 & 3.57 & 1.65 & 3.30 \\
$\quad$ Rango & $0-18$ & $0-20$ & $15-23$ & $2-17$ \\
SPIN evitación & & & & \\
Media & 5.63 & 5.03 & 22.35 & 8.48 \\
Desviación estándar & 4.10 & 4.14 & 1.86 & 4.01 \\
$\quad$ Rango & $0-26$ & $0-24$ & $18-27$ & $2-20$ \\
SPIN fisiológico & & & & \\
Media & 3.82 & 3.38 & 11.29 & 7.06 \\
Desviación estándar & 2.81 & 2.73 & 1.35 & 2.44 \\
Rango & $0-16$ & $0-16$ & $9-14$ & $3-11$ \\
\hline
\end{tabular}

Fuente: elaboración propia.

\section{Estructura interna}

Se analizaron los siguientes modelos: 1) El modelo de un factor (Ranta, et al., 2007; GarcíaLópez, et al., 2010). 2) El modelo basado en las tres subescalas teóricas (Connor, et al., 2000) en el que se proponen los siguientes factores: miedo (ítems 1, 3, 5, 10, 14 y 15), evitación (ítems 4, 6, $8,9,11,12$ y 16) y síntomas fisiológicos (ítems 2 , 7, 13 y 17). 3) El modelo de tres factores evaluado por Ranta, et al. (2007) y García-López, et al. (2010): factor 1 (ítems 1, 2, 5, 6, 7, 12, 13, 14, 15 y 17 ), factor 2 (ítems $3,4,8,10$ y 16) y factor 3 (ítems 9 y 11). 4) El modelo de cinco factores (Connor, et al., 2000): factor 1 miedo a extraños (ítems 3, 4, 8 y 10) factor 2 miedo al ridículo (ítems 5, 6, 12 y 15), factor 3 síntomas fisiológicos (ítems 2, 7, 13, 14 y 17), factor 4 miedo a la autoridad (ítems 1,14, y 16) y factor 5 miedo a hablar en público (ítems 9, 11 y 14); el ítem 14 tiene un peso factorial en los últimos tres factores.

Para conocer el ajuste de los datos a los modelos teóricos propuestos, se llevaron a cabo análisis factoriales confirmatorios (AFC). Para el análisis, se utilizó el estimador WLSMV (siglas en inglés de estimador robusto de mínimos cuadrados ponderados), que es apropiado para el AFC con datos categóricos. Este análisis se realiza sobre la matriz de correlaciones policóricas. La estimación de los modelos se efectúo con el programa Mplus 5.2 (Muthén \& Muthén, 2006).
Se considera la estimación de chi-cuadrada $\chi_{\chi}^{2}$ ) como el estadístico más utilizado para evaluar el ajuste del modelo; sin embargo, se sabe que puede ser sobreestimado cuando el tamaño de la muestra es grande o cuando existe un número muy alto de parámetros en el modelo, por lo que se sugiere ajustar dicho estadístico a los grados de libertad. En un modelo con un ajuste adecuado, se debe cumplir $\chi^{2} / g l<3$ (Bollen, 1989). También se utilizaron índices de ajuste absolutos e índices de ajuste relativos (veáse Tabla 2). Los índices de ajustes absolutos indican que el modelo propuesto reproduce los datos de la muestra, tales como el índice de bondad de ajuste (GFI), el índice de bondad de ajuste que considera los grados de libertad (AGFI) (valores $\geq 0.90$ $\mathrm{y} \geq 0.80$, respectivamente, señalan un ajuste adecuado; Hu \& Bentler, 1999), la raíz media cuadrada de los residuos (RMR) y la raíz media cuadrada del error de aproximación (RMSEA) (valores de $\leq 0.10 \mathrm{y} \leq 0.08$, respectivamente, indican un ajuste razonable; Kline, 1998). Por su parte, en los índices de ajustes relativos, se sigue la estrategia de determinar el grado en que el modelo propuesto es mejor que otro modelo alternativo (Abad, Olea, Ponsoda, \& García, 2011), tales como el indicador de Tucker Lewis (TLI), el índice de ajuste comparativo (CFI) y el índice de ajuste incremental o índice de ajuste normado (NFI). Se considera que un buen modelo debe cubrir los siguientes criterios: TLI $>0.95$, CFI > 0.95, NFI > 0.95 (Bentler, 1990; Hu \& Bentler,1999). Adicionalmente, para comparar modelos no anidados, se debe preferir el modelo asociado con el menor índice de Akaike (Brown, 2006).

El análisis final de nuestros datos señala que el modelo de tres factores de Connor et al. (2000), basado en las tres subescalas teóricas del instrumento, obtuvo los mejores indicadores en todos los aspectos. Sin embargo, estos índices se obtuvieron a partir de un ajuste en el modelo que consiste en una correlación entre la varianza del error entre ítems del factor "miedo" y el factor "evitación" (ítems 8 y 3, 10 y 4, 12 y 5). Este resultado puede deberse a que los ítems comparten la misma situación social, en la que se 
"siente miedo" o "se evita" (p. ej. el ítem 3: "tengo miedo a fiestas o eventos sociales", y el ítem 8: "evito las fiestas").

TABLA 2

Índices de bondad de ajuste para los modelos teóricos propuestos

\begin{tabular}{|c|c|c|c|c|c|c|c|c|c|c|c|}
\hline \multirow{2}{*}{ Modelo } & \multirow[b]{2}{*}{$x^{2}$} & \multirow[b]{2}{*}{$g l$} & \multirow[b]{2}{*}{$\chi^{2} / g l$} & \multicolumn{4}{|c|}{ Índices de ajuste absoluto } & \multicolumn{4}{|c|}{ Índices de ajuste relativo } \\
\hline & & & & GFI & AGFI & RMR & RMSEA & TLI & CFI & NFI & AIC \\
\hline 1 Factor & 373.06 & 66 & 5.652 & 0.92 & 0.89 & 0.05 & 0.10 & 0.94 & 0.85 & 0.39 & 454.06 \\
\hline 3 Factores ${ }^{a}$ & 167.52 & 66 & 2.538 & 0.94 & 0.92 & 0.43 & 0.06 & 0.98 & 0.95 & 0.57 & 330.90 \\
\hline 3 Factores ${ }^{b}$ & 319.42 & 65 & 4.914 & 0.91 & 0.89 & 0.61 & 0.09 & 0.95 & 0.87 & 0.87 & 423.14 \\
\hline 5 Factores & 205.32 & 63 & 3.259 & 0.94 & 0.91 & 0.05 & 0.07 & 0.97 & 0.93 & 0.55 & 364.29 \\
\hline
\end{tabular}

Nota 1: 3 factores ${ }^{a}$ (Connor, et al., 2000); 3 factores ${ }^{b}$ (Ranta, et al., 2007; García-López, et al., 2010) Nota 2: GFI = índice de bondad de ajuste; AGFI = índice de bondad de ajuste que considera los grados de libertad; RMR = raíz media cuadrada de los residuos; RMSEA = raíz media cuadrada del error de aproximación; TLI $=$ indicador de Tucker Lewis; CFI = índice de ajuste comparativo; NFI = índice de ajuste incremental; AIC =índice de Akaike.

Fuente: elaboración propia.

Análisis de confiabilidad

La confiabilidad se evalúo como consistencia interna (alfa de Cronbach) y como estabilidad temporal (correlación test-retest). En el primer caso, se encontró un nivel de confiabilidad adecuado tanto para el total de la escala $(\alpha$ $=0.959)$ como para las subescalas de miedo $(\alpha=0.908)$, evitación $(\alpha=0.910)$ y síntomas fisiológicos $(\alpha=0.825)$. Dicho análisis fue obtenido utilizando las muestras "A", "C" y "D" ( $n$ =631).

Para el cálculo de la estabilidad temporal del instrumento (evaluada a 6 semanas), se utilizaron las puntuaciones de los participantes que participaron en ambas aplicaciones (test y retest; $n=276$ ). Se obtuvieron índices adecuados, excepto en la subescala de síntomas fisiológicos. La correlación test-retest para la escala total fue $r=0.813$, para la subescala de miedo $r=0.826$, para la de evitación $r$ $=0.748$ y para la de síntomas fisiológicos $r$ $=0.656$. Las correlaciones en todos los casos fueron significativas $(p<0.001)$. Los resultados se presentan en la diagonal de la Tabla 3, el nivel de alfa se muestra en la parte superior y la correlación test-retest se indica entre paréntesis; los resultados también permiten comparar los mismos índices en las otras escalas aplicadas en el estudio.

Validez convergente y discriminante

La evidencia de validez de constructo se evaluó llevando a cabo correlaciones de Pearson entre el SPIN (y sus subescalas) y otras medidas de ansiedad social, ansiedad general y depresión. Fueron empleadas las muestras "A" y "B" (véase Tabla 3). Se considera que las puntuaciones entre 0.10 y 0.30 son débiles, entre 0.30 y 0.49 son moderadas y de 0.50 o más son fuertes (Cohen, 1988). La correlación más alta del SPIN -puntuación total- fue con el SIAS $(r=0.719)$, seguida del SPS $(r=0.675)$ y del BFNE $(r$ $=0.621)$; cada una de estas escalas también mide el constructo de ansiedad social. Por otro lado, la correlación con el BAI (ansiedad) fue de 0.430 y con el BDI (depresión) de 0.397. Estos resultados muestran niveles de validez convergente y discriminante adecuadas. 
TABLA 3

Correlaciones de Pearson entre cada uno de los instrumentos aplicados e indicadores de confiabilidad de las escalas (en la diagonal)

\begin{tabular}{|c|c|c|c|c|c|c|c|c|c|}
\hline & $\begin{array}{l}\text { SPIN } \\
\text { Total }\end{array}$ & $\begin{array}{l}\text { SPIN } \\
\text { miedo }\end{array}$ & $\underset{\text { evitación }}{\text { SPIN }}$ & $\underset{\text { fisiológico }}{\text { SPIN }}$ & BFNE & SIAS & SPS & BAI & BDI \\
\hline $\begin{array}{l}\text { SPIN } \\
\text { Total }\end{array}$ & $\begin{array}{c}0.959 \\
(0.813)\end{array}$ & & & & & & & & \\
\hline $\begin{array}{c}\text { SPIN } \\
\text { miedo }\end{array}$ & $0.908 *$ & $\begin{array}{c}0.908 \\
(0.826)\end{array}$ & & & & & & & \\
\hline $\begin{array}{c}\text { SPIN } \\
\text { evitación }\end{array}$ & $0.921^{*}$ & $0.770^{*}$ & $\begin{array}{c}0.825 \\
(0.748)\end{array}$ & & & & & & \\
\hline $\begin{array}{c}\text { SPIN } \\
\text { fisiológico }\end{array}$ & $0.791^{*}$ & $0.593^{*}$ & $0.585^{*}$ & $\begin{array}{c}0.825 \\
(0.656)\end{array}$ & & & & & \\
\hline BFNE & $0.621^{*}$ & $0.688 *$ & $0.507^{*}$ & $0.422^{*}$ & 0.892 & & & & \\
\hline SIAS & $0.719^{*}$ & $0.704^{*}$ & $0.671^{*}$ & $0.496^{*}$ & $0.632^{*}$ & 0.893 & & & \\
\hline SPS & $0.675^{*}$ & $0.658^{*}$ & $0.596^{*}$ & $0.530^{*}$ & $0.602^{*}$ & $0.596^{*}$ & 0.910 & & \\
\hline BAI & $0.430 *$ & $0.429 *$ & $0.341^{*}$ & $0.371 *$ & $0.418^{*}$ & $0.354^{*}$ & $0.563^{*}$ & 0.893 & \\
\hline BDI & $0.398^{*}$ & $0.415^{*}$ & $0.335^{*}$ & $0.290^{*}$ & $0.393^{*}$ & $0.356^{*}$ & $0.540^{*}$ & $0.393^{*}$ & 0.865 \\
\hline
\end{tabular}

Nota 1: SPIN total (puntuación total del Inventario de Fobia Social); SPIN miedo, evitación y SPIN fisiológico (subescalas del SPIN); BFNE (Escala de Miedo a la Evaluación Negativa versión breve) SIAS (Escala de Ansiedad ante la Interacción Social), SPS (Escala de Fobia Social), BAI (Inventario de Ansiedad de Beck), BDI (Inventario de Depresión de Beck). Nota 2: En la diagonal, se presenta la confiabilidad como consistencia interna (alfa de Cronbach) de todas las escalas aplicadas y entre paréntesis la correlación como estabilidad temporal (correlación testretest) para el SPIN y sus subescalas. ${ }^{*} p<0.001$

Fuente: elaboración propia.

\section{Poder discriminativo}

Se realizó un análisis de curvas ROC (siglas en inglés de características operativas del receptor), con el propósito de evaluar la capacidad de discriminación de la puntuación total del SPIN como criterio de decisión para establecer un diagnóstico de trastorno de ansiedad social. Para cada punto de corte (diagnóstico de ansiedad social a partir de los criterios del DSM-5 realizado por el médico psiquiatra), se calcularon los siguientes indicadores: sensibilidad (capacidad del SPIN para detectar correctamente pacientes con ansiedad social de pacientes con ansiedad generalizada), especificidad (capacidad del SPIN para identificar correctamente pacientes que no presentan ansiedad social pero sí ansiedad generalizada), valor predicho positivo (o probabilidad de que un paciente identificado por el SPIN con ansiedad social la presente realmente), valor predicho negativo (o probabilidad de que un paciente identificado por el SPIN sin ansiedad social en realidad no la presente).

Los resultados señalan que el área bajo la curva ROC fue de 0.958 (95 \% IC, 0.95-1), significativo $(p<0.001)$. Estos resultados sugieren que existe 95\% de probabilidad de que una persona con trastorno de ansiedad social tenga una puntuación más alta en el SPIN de lo que tendría una persona con trastorno de ansiedad generalizada.

Aun cuando el área bajo la curva es el índice más ampliamente utilizado para evaluar la capacidad diagnóstica de un instrumento, el índice Youden (1950) es otra medida importante para evaluar la eficiencia. Este índice provee de un criterio para elegir el umbral óptimo, por tanto, la elección del punto de corte se basa en este indicador.

La Tabla 4 muestra, para algunos puntos de corte elegidos, la sensibilidad, la especificidad, el valor predicho positivo, el valor predicho negativo, la razón de verosimilitud negativa y el índice de Youden. Los resultados muestran que un punto de corte de 25 produce el mejor balance, ya que cuenta con una buena sensibilidad y especificidad (70.8 y $100 \%$, respectivamente), un valor predicho positivo de $100 \%$, un valor predicho negativo de $58 \%$ y un índice de Youden correspondiente a 0.95. Se sugiere que un punto de corte adecuado debe tener una sensibilidad mínima de 0.70 y una especificidad mínima de 0.80 . 


\section{TABLA 4}

Para puntos de corte seleccionados se muestra la sensibilidad, la especificidad, el valor predicho positivo, el valor predicho negativo, el índice de Youden y la razón de verosimilitud negativa

\begin{tabular}{ccccccc}
\hline $\begin{array}{c}\text { Puntode } \\
\text { corte }\end{array}$ & Sensibilidad & Especificidad & PPV & NPV & $\begin{array}{c}\text { Indice de } \\
\text { Youden }\end{array}$ & LR- \\
\hline 34 & 0.911 & 1 & 1 & 0.90 & 0.569 & 0.089 \\
30 & 0.810 & 1 & 1 & 0.76 & 0.902 & 0.190 \\
25 & 0.708 & 1 & 1 & 0.58 & 0.950 & 0.292 \\
21 & 0.638 & 1 & 1 & 0.42 & 0.840 & 0.363 \\
19 & 0.614 & 1 & 1 & 0.36 & 0.760 & 0.386 \\
17 & 0.593 & 1 & 1 & 0.30 & 0.660 & 0.407 \\
15 & 0.560 & 1 & 1 & 0.20 & 0.580 & 0.440 \\
13 & 0.543 & 1 & 1 & 0.14 & 0.520 & 0.457 \\
\hline
\end{tabular}

Nota: PPV (valor predicho positivo), NPV (valor predicho negativo), LR(razón de verosimilitud negativa).

Fuente: elaboración propia.

\section{Discusión}

El objetivo de este trabajo fue analizar las propiedades psicométricas del Inventario de Fobia Social (SPIN), debido a la ausencia en nuestro país de una validación de dicho instrumento (el cual es uno de los más usados para la evaluación del trastorno de ansiedad social).

Las puntuaciones obtenidas por las muestras de estudiantes mexicanos fueron menores a las reportadas en otros estudios para universitarios canadienses -tanto de habla inglesa como de francesa- (Radomsky, et al., 2006) y para adolescentes brasileños (Vilete, et al., 2004). Sin embargo, el valor de la media fue cercano al hallado en adolescentes finlandeses (Ranta, et al., 2007) y españoles (García-López, et al., 2010). Con respecto a los sujetos con trastorno de ansiedad social, nuestros datos indican una media más elevada que la reportada en la publicación original con individuos estadounidenses (Connor, et al., 2000) y en lo publicado con muestras canadienses (Antony, et al., 2006) y alemanas (Sosic, et al., 2008).

La menor puntuación reportada por los estudiantes mexicanos pudiera deberse a un efecto de "deseabilidad social", concepto que se refiere a la necesidad de aprobación y aceptación social, con la creencia de que esta puede obtenerse por medio de conductas culturalmente apropiadas y aceptables (Marlow \& Crowne, 1961). La deseabilidad social es considerada como una parte normal del desarrollo y, generalmente, disminuye a medida que va aumentando la edad (Klesges et al., 2004). Nótese que se ha observado en muestras mexicanas mayores niveles de deseabilidad social respecto del nivel encontrado en otros países (p. ej. Eysenck \& Lara-Cantú, 1989; Moral, 2007).

En lo que concierne a la estructura interna del SPIN, la publicación original sugiere una estructura teórica de tres factores basada en las subescalas del instrumento; pero, en dicho estudio, de manera empírica, se obtuvo una solución de 5 factores (Connor et al., 2000). Por su parte, otros estudios, como el llevado a cabo por Ranta et al. (2007), y evaluado posteriormente por García-López, et al. (2010), han llegado a proponer tanto una estructura alternativa de 3 factores como una solución unifactorial. En lo que respecta a los resultados de nuestra investigación, los índices de bondad de ajuste sugieren que la solución de tres factores basada en las subescalas del instrumento (Connor et al., 2000) es la mejor, siempre y cuando se tenga en cuenta la covariación entre los errores de los ítems que pertenecen a factores distintos. Esto, sin duda, no es adecuado, por lo que se sugiere una modificación en las situaciones que se evalúan para que los factores sean más claros; además, la correlación entre los factores latentes es muy alta (arriba de 0.86), lo cual pudiera sugerir una solución unifactorial.

La consistencia interna que mostró el SPIN fue excelente, y también fue muy buena en sus tres subescalas. Esta alta consistencia interna es superior a la hallada en la publicación original para el total de la escala (Connor et al., 2000) y en lo reportado en otros estudios (Radomsky et al., 2006; Vilete et al., 2004; Ranta et al., 2007; Antony et al., 2006; Sosic et al., 2008; Osório et al., 2009; García-López et al., 2010).

Los ítems que obtuvieron una media mayor fueron el 11 y el 15 , mientras que en el 3 y el 16 se observaron las medias más bajas. Es decir que, en las muestras analizadas, los individuos refieren evitar frecuentemente dar discursos (ítem 11) y experimentar fácilmente miedo a 
hacer el ridículo o parecer estúpido (ítem 15). Sin embargo, ante las fiestas y los acontecimientos sociales no refieren experimentar mucho temor (item 3), y niegan que eviten frecuentemente hablar con alguien que tenga autoridad (ítem 16). Debe destacarse que tanto en esta investigación como en la publicación original (Connor et al., 2000) y en otros estudios (Ranta et al., 2007; Osório et al., 2009), el ítem que puntuó más alto fue el 11 ("evito dar discursos"). Aportandose así evidencia a lo ya dicho por otros autores (Caraveo \& Colmenares, 2000; Furmark, 2002; Furmark, Tillfors, Stattin, Ekselius, \& Fredrikson, 2000; Stein \& Deutsch, 2003) de que hablar en público es la situación social más temida tanto por personas con trastorno de ansiedad social como por la población en general.

El SPIN presentó una buena confiabilidad testretest en un periodo de 6 semanas, tanto en la escala total como en cada una de sus subescalas. Esto coincide con la buena confiabilidad testretest presentada en el estudio original para el total de la escala (Connor et al., 2000) y en lo expuesto por Antony et al. (2006), Radomsky et al. (2006) y Ranta et al. (2007).

Se realizaron correlaciones entre el SPIN y otras medidas. Las correlaciones más elevadas que se obtuvieron, fueron con las escalas que también miden ansiedad social (SIAS, SPS y BFNE), lo cual coincide con lo hallado en otros estudios (Antony et al., 2006; Socic et al., 2008), donde se han reportado correlaciones altas con el SIAS y el SPS. A su vez, como era de esperarse, se encontraron correlaciones más bajas con otras escalas que miden constructos diferentes (validez discriminante). La correlación con el BAI que mide la ansiedad de forma general resultó moderada, lo cual era esperable, ya que evidentemente la ansiedad social es un trastorno de ansiedad. Con respecto al BDI que evalúa depresión, la correlación fue menor (pero también resultó positiva y significativa), al igual que lo hallado en otros estudios (Radomsky et al., 2006; Sosic et al., 2008), lo cual se explica por la frecuente comorbilidad existente entre ansiedad social y depresión (Acarturk et al., 2008; Chartier, et al., 2003). Así, puede concluirse que la escala presenta una adecuada validez convergente y discriminante.

Una de las fortalezas del SPIN es su capacidad para identificar personas que presentan trastornos de ansiedad de las que pertenecen a población en general (GarcíaLópez et al., 2010). Además, en nuestro estudio, se demuestra que el instrumento es capaz de diferenciar adecuadamente el trastorno de ansiedad social del trastorno de ansiedad generalizada. En la población estudiada, una puntuación de 25 se considera como el mejor punto de corte, lo cual es cercano a los 24 puntos sugeridos en población finlandesa (Ranta et al., 2007).

De esta manera, tomando en cuenta los resultados obtenidos, se puede concluir que la versión en español del SPIN es una medida válida y confiable para la evaluación del trastorno de ansiedad social en las muestras mexicanas estudiadas.

Existen algunas limitaciones que podrían dificultar la generalización de las conclusiones del presente estudio. En primer lugar, la mayoría de los participantes consistieron en estudiantes universitarios de sexo femenino, por lo que en futuros estudios sería conveniente tener muestras equilibradas en género. A la vez, sería deseable contar con muestras de población general y así poder evaluar las propiedades psicométricas de la escala en personas con un mayor rango de variables sociodemográficas. Respecto de la aplicación en las muestras clínicas, debe mencionarse que no se utilizó ningún instrumento $\mathrm{u}$ otro evaluador para confirmar el diagnóstico (como hubiera sido deseable). Por último, también sería conveniente evaluar la sensibilidad del instrumento al cambio clínico después de un tratamiento terapéutico.

\section{Agradecimientos}

No se contó con financiamiento para la realización de la investigación. Los autores manifiestan no tener ningún tipo de conflicto de intereses con respecto al artículo. Agradecemos al Dr. Esteban Gudayol Ferré por sus valiosas 
Marcelo-Archibaldo Bravo, Fabiola González Betanzos, Álvaro Castillo Navarro, Et al.

aportaciones, las cuales permitieron mejorar una versión preliminar del presente estudio.

\section{Referencias}

Abad, F., Olea, J., Ponsoda, V., \& García, C., (2011). Medición en ciencias sociales y de la salud. Madrid: Editorial Síntesis.

Acarturk, C., Graaf, R., Straten, A., Have, M. T., \& Cuijpers, P. (2008). Social phobia and number of social fears, and their association with comorbidity, healthrelated quality of life and help seeking. Social Psychiatry and Psychiatric Epidemiology, 43(4), 273-279.

Aderka, I. M., Hofmann, S. G., Nickerson, A., Hermesh, H., Gilboa-Schechtman, E., \& Marom, S. (2012). Functional impairment in social anxiety disorder. Journal of Anxiety Disorders, 26(3), 393-400.

Allgulander, C., Mangano, R., Zhang, J., Dahl, A. A., Lepola, U., Sjodin, I., \& Emilien, G. (2004). Efficacy of venlafaxine ER in patients with social anxiety disorder: A double-blind, placebo-controlled, parallelgroup comparison with paroxetine. Human Psychopharmacology, 19(6), 387-396.

American Psychiatric Association (2013). Diagnostic and statistical manual of mental disorders (5th ed.). Arlington, VA: American Psychiatric Publishing.

Antony, M. M., Coons, M. J., McCabe, R. E., Ashbaugh, A., \& Swinson, R. P. (2006). Psychometric properties of the social phobia inventory: Further evaluation. Behaviour Research and Therapy, 44, 1177-1185.

Bentler, P. M. (1990). Comparative fit indexes in structural models. Psychological Bulletin, 107, 238-246.

Bernaldo-de-Quirós, M., Estupiñá, F. J., Labrador, F. J., Fernández-Arias, I., Gómez, L., Blanco, C., \& Alonso, P. (2012). Diferencias sociodemográficas, clínicas y de resultados del tratamiento entre los trastornos de ansiedad. Psicothema, 24(3), 396-401.

Bollen, K. A. (1989). Structural equations with latent variables. Nueva York: Wiley.
Bravo, M. A., González Betanzos, F., Castillo Navarro, A. \& Padrós Blázquez, F. (2015). Escala de miedo a la evaluación negativa versión breve (BFNE): propiedades psicométricas en muestras clínicas y de universitarios en México. Acta Colombiana de Psicología, 18(1), 69-77.

Brown, T. A. (2006). Confirmatory factor analysis for applied research. Nueva York: Guilford.

Caraveo, J., \& Colmenares, E. (2000). Prevalencia de los trastornos de ansiedad fóbica en la población adulta de la ciudad de México. Salud Mental, 23(5), 10-19.

Chartier, M. J., Walker, J. R., \& Stein, M. B. (2003). Considering comorbidity in social phobia. Social Psychiatry and Psychiatric Epidemiology, 38(12), 728-734.

Cohen, J. (1988). Statistical power analysis for the behavioral sciences (2.․ ed.). Hillsdale, NY: Lawrence Erlbaum Associated.

Connor, K. M., Davidson, J. R. T., Churchill, L. E., Sherwood, A., Foa, E., \& Weisler, R. H. (2000). Psychometric properties of the Social Phobia Inventory (SPIN). British Journal of Psychiatry, 176, 379-386.

Davidson, J. R. T., Potts, N. L. S., Richichi, E. A., Krishnan, R., Ford, S. M., Smith, R. D., \& Wilson, W. (1991). The Brief Social Phobia Scale. Journal of Clinical Psychiatry, 52, 48-51.

Eysenck, S. B. G., \& Lara-Cantú, M. A. (1989). Un estudio transcultural de la personalidad en adultos mexicanos e ingleses. Salud Mental, 12(3), 14-20.

Furmark, T. (2002). Social phobia: Overview of community surveys. Acta Psychiatrica Scandinavica, 105(2), 84-93.

Furmark, T., Tillfors, M., Stattin, H., Ekselius, L., \& Fredrikson, M. (2000). Social phobia subtypes in the general population revealed by cluster analysis. Psychological Medicine, 30(6), 1335-1344.

García-López, L. J., Bermejo, R. M., \& Dolores-Hidalgo, M. (2010). The Social Phobia Inventory: Screening and crosscultural validation in Spanish adolescents. The Spanish Journal of Psychology, 13(2), 970-980. 
García-Portilla, M. P., Bascarán, M. T., Sáiz, P. A., Parellada, M., Bousoño, M., \& Bobes, J. (2008). Banco de instrumentos básicos para la práctica de la psiquiatría clínica. España: Ars Medica.

Hu, L., \& Bentler, P. M. (1999). Cutoff criteria for fit indexes in covariance structure analysis: Conventional criteria versus new alternatives. Structural Equations Modeling, 6, 1-55.

Jurado, S., Villegas, M. E., Méndez, L., Rodríguez, F., Loperena, V., \& Varela, R. (1998). La estandarización del Inventario de Depresión de Beck para los residentes de la ciudad de México. Salud Mental, 21(3), 26-31.

Klesges, L. M., Baranowski, T., Beech, B., Cullen, K., Murray, D. M., Rochon, J., \& Pratt, C. (2004). Social desirability bias in self-reported dietary, physical activity and weight concerns measures in 8- to 10 year-old African-American girls: Results from the Girls Health Enrichment Multisite Studies (GEMS). Preventive Medicine, 38, 78-87.

Kline, R. B. (1998). Principles and practice of structural equation modeling. Nueva York: Guilford.

Labrador, F. J., \& Ballesteros, F. (2011). Efectividad de los tratamientos para la fobia social en el ámbito aplicado. Psicothema, 23(4), 560-565.

Leary, M. R. (1983). A brief version of the Fear of Negative Evaluation Scale. Personality and Social Psychology Bulletin, 9, 371-375.

Marlow D., \& Crowne D. P. (1961). Social desirability and response to perceived situationals demands. Journal of Consulting and Clinical Psychology, 25(2), 109-115.

Mattick, R. P., \& Clarke, J. C. (1998). Development and validation of measures of social phobia scrutiny fears and social interaction anxiety. Behaviour Research and Therapy, 36, 455-470.

Medina-Mora, M. E., Borges, G., Lara, C., Benjet, C., Blanco, J., Fleiz, C., ... \& Aguilar-Gaxiola, S. (2003). Prevalencia de trastornos mentales y uso de servicios: resultados de la encuesta nacional de epidemiología psiquiátrica en México. Salud Mental, 26(4), 1-16.

Moral, J. (2007). Control de la deseabilidad social ante la evaluación del ajuste diádico y la satisfacción marital. Psicología y Ciencia Social, 9(2), 5-12.

Muthén, L. K., \& Muthén, B. O. (2006). Mplus user's guide (Version 4). Los Ángeles, CA: Muthén \& Muthén.

Ohayon, M. M., \& Schatzberg, A. F. (2010). Social phobia and depression: Prevalence and comorbidity. Journal of Psychosomatic Research, 68(3), 235-243.

Osório F. L., Crippa, J. A., \& Loureiro, S. R. (2009). Cross-cultural validation of the Brazilian Portuguese version of the Social Phobia Inventory (SPIN): Study of the ítems and internal consistency. Revista Brasileira de Psiquiatria, 31 (1), 25-29.

Radomsky, A. S., Ashbaugh, A. R., Saxe, M. L., Ouimet, A. J., Golden, E. R., Lavoie, S. L., \& O'Connor, K. P. (2006). Psychometric properties of the French and English versions of the Social Phobia Inventory. Canadian Journal of Behavioural Science, 38(4), 354-360.

Ranta, K., Kaltiala-Heino, R., Koivisto, A. M., Tuomisto, M. T., Pelkonen, M., \& Marttunen, M. (2007). Age and gender differences in social anxiety symptoms during adolescent: The social phobia inventory (SPIN) as a measure. Psychiatry Research, 153, 261-270.

Rickels, K., Mangano, R., \& Khan, A. (2004). A double-blind, placebo-controlled study of a flexible dose of venlafaxine ER in adult outpatients with generalized social anxiety disorder. Journal of Clinical Psychopharmacology, 24(5), 488-496.

Robles, R., Varela, R., Jurado, S., \& Páez (2001). Versión mexicana del Inventario de Ansiedad de Beck: propiedades psicométricas. Revista Mexicana de Psicología, 18(2), 211-218.

Simon, N. M., Otto, M. W., Korbly, N. B., Peters, P. M., Nicolaou, D. C., \& Pollack, M. H. (2002). Quality of life in social anxiety 
disorder compared with panic disorder and the general population. Psychiatric Services, 53(6), 714-718.

Somers, J. M., Goldner, E. M., Waraich, P., \& Hsu, L. (2006). Prevalence and incidence studies of anxiety disorders: A systematic review of the literature. Canadian Journal of Psychiatry, 51(2), 100-113.

Sosic, Z., Gieler, U., \& Stangier, U. (2008). Screening for social phobia in medical inand outpatients with the German version of the social phobia inventory (SPIN). Journal of Anxiety Disorders, 22, 849-859.

Stein, M. B., \& Deutsch, R. (2003). In search of social phobia subtypes: Similarity of feared social situations. Depression and Anxiety, 17(2), 94-97.

Stein, D. J., Versiani, M., Hair, T., \& Kumar, R. (2002). Efficacy of paroxetine for relapse prevention in social anxiety disorder: A 24week study. Archives of General Psyquiatry, 59, 1111-1118.

Torgrud, L. J., Walker, J. R., Murray, L., Cox, B. J., Chartier, M., \& Kjernisted, K. D. (2004). Deficits in perceived social support associated with generalized social phobia. Cognitive Behaviour Therapy, 33(2), 87-96.

Vilete, L. M., Coutinho, E. S., \& Figueira, I. L. (2004). Confiabilidade da versão em Português do Inventário de Fobia Social (SPIN) entre adolescentes estudantes do Municipio do Rio de Janeiro. Cad. Saúde Pública, 20(1), 89-99.

Watson, D., \& Friend, R. (1969). Measurement of social-evaluative anxiety. Journal of Consulting and Clinical Psychology, 33, 448-457.

Wong, N., Sarver, D. E., \& Beidel, D. C. (2012). Quality of life impairments among adults with social phobia: The impact of subtype. Journal of Anxiety Disorders, 26(1), 50-57.

Youden, W. J. (1950). Index for rating diagnostic test. Cancer, 3, 32-35.

\section{Notas}

* Artículo de investigación. 